\title{
Mediation Effect of Perception about School Climate on the Relationship Between Students' Achievement Motivation and Academic Performance
}

\author{
Jinping Li \\ College of Education, Northwest Normal University, Lanzhou 730070, Gansu, China
}

\begin{abstract}
The purpose of this study is to explore the mediating effect of school climate perception on the relation between achievement motivation (including SOAM and IOAM) and academic performance. 324 valid questionnaires from middle school students were used to analyze. Correlation analysis results show that there is a significant negative correlation between academic performance and SOAM, but academic performance positively correlated with IOAM. Furthermore, the perception of study pressure, school order and discipline are negatively correlated with academic performance, while the perception of teacher-student relationship, classmate relationship and the development of diversity are positively correlated with academic performance. Mediation analysis show that there is a partial mediating effect of the study pressure perception on the relationship between SOAM, IOAM and academic performance. Findings from this study suggest that school education should guide students to clarify their learning goals and reduce external study pressure, which helps to promote their academic performance.
\end{abstract}

Keywords: Achievement motivation, Study pressure, Academic performance, Mediating effect.

\section{Introduction}

Students' academic performance, especially in Chinese, Mathematics and English, can predict their learning opportunities in the future. In general, students with good academic performance can get more learning opportunities. Furthermore, students' academic performances were correlated with their mental health, social adaption, interpersonal relationship, and self-esteem (Zhang, Qin, \& Ren, 2018; Bond, Chykina, \& Jones, 2017). Therefore, it is important for researchers to find factors affecting learners' academic performance. Previous studies indicated that students' academic performances were influenced by internet addiction (Zhang, Qin, \& Ren, 2018), academic engagement (Jelas, Azman, Zulnaidi, \& Ahmad, 2016), and social network (Bond, Chykina, \& Jones, 2017).

Although learners' academic performance is determined by a variety of factors, "why learners study" is still related to motivation (Viljaranta, Aunola, \& Hirvonen, 2016). Motivation has been described as the intensity of behavior, the direction of behavior, and the duration of behavior (Ames, 1990). For learners, motivation was related to their learning purpose, learning activities initiation, and maintain of learning behavior. Individuals with different motivational orientations have different learning behaviors and academic performance may be different. Some students' learning behaviors are driven by the approval and praises from significant others (e.g. parents, teachers), while some students are inspired by their own interests. According to Dweck's $(1986,2006)$ theoretical approach, the circumscribed goal or orientation are people's achievement motivation, and the 2 (mastery, performance) $\times 2$ (approach, avoidance) achievement goal framework was divided into approach or avoidance goals. Striving to master new tasks and the approach of success refers to mastery-approach goals (MAP), whereas mastery-avoidance goals (MAV) focus on the avoidance of task-based or intrapersonal incompetence. Moreover, performanceapproach goals (PAP) refers to people striving to demonstrate high ability and validate their competence in relation to others, whereas performance-avoidance goals (PAV) are strategies that prevent the individual from showing a lack of competence or obtain unfavorable judgments from others (Bipp, \& van Dam, 2014).

In cross-cultural researches, researchers found that peoples' achievement motivations were influenced by the sociocultural contexts (Mcinerney, Maehr, \& Dowson, 2004; Tao, \& Hong, 2000, 2013). Compared with Western culture, Eastern Asian culture tend to collectivism, and individuals' behavior is more likely to be influenced by the opinions of others (Briley, Wyer, $\& \mathrm{Li}, 2014)$. In Chinese culture, achievement motivation can not only motivate learners to learn new knowledge, but also help them gain acceptance and respect from others. Yang and $\mathrm{Yu}$ (1988) divided achievement motivation into Individual-Oriented Achievement Motivation (IOAM) and Social-Oriented Achievement Motivation (SOAM). The former refers to the desire to achieve goals that are defined by individuals themselves; while the latter was defined as the desire to achieve goals defined by significant others ( $\mathrm{Yu} \&$ Yang, 1994). In the present study, we will attempt to compare the predictive effects of IOAM and SOAM on academic performance, and assume that there was a difference of their impact on academic performance.

The difference in students' orientation affect their focus on external environmental information. According to the goal perspectives theory (Roberts, 2001), individual goal orientation affects their thinking, feelings and actions. For example, learners with communicate mastery or task-oriented goal perspective value the process of learning itself, try hard to learn new skills and to develop personal competence without the need of external rewards or threats; while, learners with ego-oriented goal perspective feel successful and satisfied when they attain higher performance than their group of reference (Theodosiou, \& Papaioannou, 2006). Therefore, there is a possibility that students with SOAM are more concerned with school environmental factors, such as 
teacher-student relationships, classmate relationships, etc., while IOAM may tend to pay less attention to relevant information. These difference of perception about external information (i.e. school climate and parental expectation) will in turn affect students' academic performance (Englund, Luckner, Whaley, \& Egeland, 2004; Wang \& Holcombe, 2010). In the school educational context, students' school climate perception relates to their learning behavior and learning achievement (Amemiya, \& Wang, 2018). For example, Cohen, Garcia and Goyer (2017)found that perception of supportive school climate would positively influence academic achievement for the African American students; on the contrary, a poor perceived school climate might lead to student social withdrawal and other negative consequences, such as school dropout (Nickerson, Singleton, Schnurr, \& Collen, 2014; Wachs, 2012).

School climate is defined as shared beliefs, values, and attitudes that shape interactions between students, teachers, and administrators and set the parameters of acceptable behavior and norms for the school (Kuperminc, Leadbeater, Emmons, \& Blatt, 1997). It is a multiple construction that include caring relationships with adults, safety, school connectedness, and meaningful participation (Cohen et al., 2009; Koth, Bradshaw, \& Leaf, 2008; Modin \& Östberg, 2009; Thapaet al., 2013; Zullig et al., 2010), and De Pedro, Gilreath and Berkowitz (2016) beleived that caring relationships is a student's perception of the extent of social and emotional support they receive from teachers and other school adults. Safety is a student's sense of physical and social-emotional security. School connectedness is the degree to which students feel they belong to a school community and have a positive connection with peers and adults in a school community. Meaningful participation is the involvement of students in relevant, engaging and interesting opportunities that develop a sense of responsibility in the school community. However, Ge and Yu (2006) thought that the effect of school climate varied with the individual because different people have different perceptions of it, and they divided the school climate into five dimensions: teacher-student relationship, classmate relationship, study pressure, school order and discipline, and the development of diversity. Teacher-student relationship is defined as the psychological relationship between teachers and students, including care, respect, trust, equality, fairness, etc. Classmate relationship is the psychological relationship in the process of interaction between students in the school, such as mutual help, honesty, friendship, respect, humility, etc. Study pressure is defined as the subjective feelings of pressure from learning tasks, study schedules, and so on. School order and discipline refers to the orderliness of school life, and the controlling over students' bad behavior. The development of diversity is defined as the richness of students' school life, and relevant measures taken by the school to promote the all-round development of students. In fact, the effect of the school climate on students is determined by their perception for it. That is, student personal experiences of the school climate mediate actual school climate effects (Kuperminc, Leadbeater, \& Blatt, 2001; Loukas \& Robinson, 2004). Wang and Holcombe (2010) found that students' perceptions of school environment influenced their academic achievement directly. In this study, the Perceived School Climate Scale (PSCS) developed by Ge and $\mathrm{Yu}$ (2006) will be applied to measure the middle school students' perception of school climate, and explore its effect on academic performance. We hypothesize that students' perceptions of teacher-student relationship, classmate relationship, and study pressure, the development of diversity, school order and discipline have different effects on students' academic performances. The effects from teacher-student relationship, classmate relationship and the development of diversity are positive, while the impacts of study pressure, school order and discipline are negative.

Students' perception of the school climate affects directly their learning achievement, but also mediates the influence of achievement motivation on academic performance. Researches have shown that students who feel warm and supportive relationship from their teachers tend to report high levels of engagement and interest in academics (Ryan \& Patrick, 2001), and positive classroom community influences their motivational dimensions, such as achievement goals (Nelson \& DeBacker, 2008) and interest (Wentzel, Battle, Russell, \& Looney, 2010); while negative peer climate declines students' autonomous motivation in mathematics (Reindl, Berner, Scheunpflug, Zeinz, \& Dresel, 2015). Moreover, there are a series of studies revealed that a positive school climate increased student attendance in middle school (Purkey \& Smith, 1983; Sommer, 1985), and it has a powerful influence on the motivation to learn (Thapa, \& Cohen, 2013). As mentioned above, achievement motivation orientation mainly includes IOAM and SOAM under Chinese cultural context (Yang \& Yu, 1988), and their influence on academic performance are different from approach or avoidance goals (Bipp, \& van Dam, 2014). Furthermore, China's school climates encourages the construction of a social reality of close interdependence, enduring group membership (Bush, \& Haiyan, 2000; Wagner, 2002). Therefore, students with different motivation orientations have different perception about school climate, while the effect of motivation orientations on academic performance is mediated by students' perception of school climate. In the present study, we assumed that there is a mediation effect of school climate perception on the relationship between the achievement motivations and academic performance.

\section{Research Method}

\subsection{Participants}

A stratified sampling method was adopted in order to explore the relationship between students' perceptions of school climate, learning motivation and their academic performance. 330 students from 12 boarding middle school were recruited to answer questionnaires in the present study, 6 questionnaires were deleted because of incomplete. A total of 324 valid questionnaires were used to analysis. This sample included $161(49.7 \%)$ boys and 163 (50.3\%). They were between 13 and 17 years old, and average age was $15.49(\mathrm{SD}=1.25)$, and distributed over three school grades - 110 in 7 th grade students $(\mathrm{M}=14.3 ; \mathrm{SD}=0.64 ; 48.2 \%$ male $) ; 107$ in 8 th graders $(\mathrm{M}=15.6 ; \mathrm{SD}=0.72 ; 53.3 \%$ male); and 107 in 11 th graders $(\mathrm{M}=16.6 ; \mathrm{SD}=0.71 ; 47.7 \%$ male $)$. All participants have provided written informed consent before enrolment, and all procedures were approved by the ethical committee at College of Education, Northwest Normal University. 


\subsection{Measures}

The Perceived School Climate Scale (PSCS, Ge \& Yu, 2006) was used to assess level of students' perception about their school climate. It was specifically based on school ethos aspects, and was consisted of 38 items grouped into teacher-student relationship(e.g., "Our teachers could trust deeply their students." $\alpha=0.85$ ), classmate relationship (e.g., "Classmates could support and encourage each other." $\alpha=0.75$ ), study pressure (e.g., "The teacher did not pressure students to learning." $\alpha=0.71$ ), the order and discipline (e.g., "The management of classroom discipline was greatly effective." $\alpha=0.68$ ), and the development of diversity(e.g., "Our classmates are versatile." $\alpha=0.7$ ) scales. All of sub-scales have high reliability and validity, and the total scale presents a good Cronbach's alpha coefficient of .82 in the present study, indicating good internal consistency. Every item was answered on four-point Likert scales from 1 (strongly disagree) to 4 (strongly agree), In addition to academic pressure, the higher scores indicate more positive perceptions of school climate.

Achievement Motivation Scale (AMS; Yu, 1990) is a 13-item scale designed to assess students' achievement motivation. According to the $\mathrm{Yu}$ (1990), achievement motivation should be divided two aspects: social oriented achievement motivation (SOAM; e.g. "The major goal in my life is to work hard to achieve something which will make my parents feel proud of me.") and individual oriented achievement motivation (IOAM; e.g. "No matter how many times I fail, I'll keep on trying even without others' encouragement."). This scale includes 60 items about $\operatorname{SOAM}(30)$ and $\operatorname{IOAM}(30)$, and all items are rated on the following four-point scale: $1=$ strongly disagree, $2=$ disagree, $3=$ =general, $4=$ agree, $4=$ strongly agree. The higher scores indicate the higher level of achievement motivation. e. Yu and Yang (1994) achieved alphas of .89 for both sub-scales. In the current study, Cronbach's as for two sub-scales (such as SOAM, IOAM) and total scale were $.83, .69$ and .77 .
Academic achievement. In this study, we used academic standardized school district achievement tests to collected test scores for students in three courses, such as Chinese, Mathematics and English. These tests are designed and compiled by the school district according to the standardized syllabus, and cover the knowledge points of each subject. They are often used to assess students' level of learning.

\subsection{Data Analyses}

Descriptive statistics and correlational analysis were performed by using the IBM ${ }^{\circledR}$ SPSS ${ }^{\circledR}$ Statistical package, version 20 computer software, including mean, standard deviation, frequency, and percentage were utilized to describe the characteristics of the participants. Mediation analysis was carried out using Mplus 7.0 to examine whether students' perception of school climate mediated the relationship between achievement and academic performance. To evaluate the overall goodness-of-fit of the model, chi-square tests, the Tucker-Lewis Index (TLI), the Comparative Fit Index criterion (CFI) and the Root Mean Square Error of Approximation (RMSEA) were used (Hu \& Bentler, 1999). For the TLI and CFI, the lower limits were 0.9. A TLI or CFI value equal to or exceeding 0.95 was considered indicative of high confidence of fit. A RMSEA value smaller than 0.08 was considered indicative of an adequate model fit (Vandenberg \& Lance, 2000). The standardized coefficients, standard errors and $95 \%$ confidence intervals were reported.

\section{Results}

\subsection{Correlational Analysis}

The normality of all variables was calculated, and the values of skew and kurtosis of variables in the mediation model ranged from -0.65 to $0.41,-0.58$ to 0.71 , respectively. The means, standard deviations, and correlation coefficients between LAA, SOAM, IOAM, and the five dimensions of PSC are presented in Table 1.

Table 1: All variables' means, standard deviations, and correlation matrix $(\mathrm{N}=324)$.

\begin{tabular}{|c|c|c|c|c|c|c|c|c|c|c|}
\hline & $M$ & $S D$ & PRTS & PRCS & $P S P$ & $P S O D$ & $P D D$ & $S O A M$ & $I O A M$ & $L A A$ \\
\hline PRTS & 3.150 & 0.605 & - & & & & & & & \\
\hline PRCS & 2.974 & 0.515 & $.662 * *$ & - & & & & & & \\
\hline PSP & 2.317 & 0.531 & $-.480 * *$ & $-.330 * *$ & - & & & & & \\
\hline PSOD & 2.620 & 0.317 & -.073 & -.030 & $.333^{* *}$ & - & & & & \\
\hline PDD & 2.971 & 0.571 & $.696^{* *}$ & $.659^{* *}$ & $-.376^{* * *}$ & -.021 & - & & & \\
\hline SOAM & 2.827 & 0.607 & -.010 & -.037 & $.174^{* *}$ & $.242^{* *}$ & .004 & - & & \\
\hline IOAM & 3.934 & 0.769 & $.571 * *$ & $.446^{* *}$ & $-.397 * *$ & $-.150 * *$ & $.471 * *$ & -.070 & - & \\
\hline LAA & 62.075 & 16.853 & $.276^{* *}$ & $.143^{* *}$ & $-.280^{* * *}$ & $-.125^{*}$ & $.187^{* *}$ & $-.164 * *$ & $.352^{* * *}$ & - \\
\hline
\end{tabular}

Note: **. Correlation is significant at the 0.01 level (2-tailed).*. Correlation is significant at the 0.05 level (2-tailed). PRTS is the perception of teacher-student relationship; PRCS is the perception of classmate relationship; PSP is the perception of study pressure; PSOD is the perception of school order and discipline; PDD is the perception of the development of diversity, LAA refers to academic performance.

Pearson correlation analysis results showed that LAA was negatively correlated with PSP, PSOD and SOAM; and positively correlated with PRTS, PRCS, PDD and LOAM. Furthermore, there were significant positive correlations between SOAM and PSP, PSOD. While there were significant positive correlations between LOAM and PRTS, PRCS; and significant negative correlations between LOAM and PSP, PSOD.

\subsection{Mediation Analysis}

Mediation analysis was carried out using Mplus 7.0 (Muthén and Muthén, 2012) to examine whether students' perception about school climate mediated the relationship between achievement motivation and LAA. According to the hypothesis, LOAM and SOAM were the independent variable, the perception about teacher-student relationship, classmate relationship, academic pressure, school order and discipline, 
and the development of diversity were the mediator variables, and LAA was the dependent variable. The mediation analysis of the initial model showed that PRTS, PRCS, PSOD and PDD have not significant effects on students' LAA, but PSP and SOAM were predicted negatively LAA, and IOAM were predicted positively LAA. The model failed to obtain sufficient goodness-of-fit: $\chi 2=375.846, \mathrm{df}=10, \chi 2 / \mathrm{df}=37.585$, $\mathrm{p}=0.000 ; \mathrm{TLI}=-0.313 ; \mathrm{CFI}=0.531 ; \mathrm{RMSEA}=0.337$ (90\% CI $[0.308,0.366] ; \mathrm{p}=0.013)$.

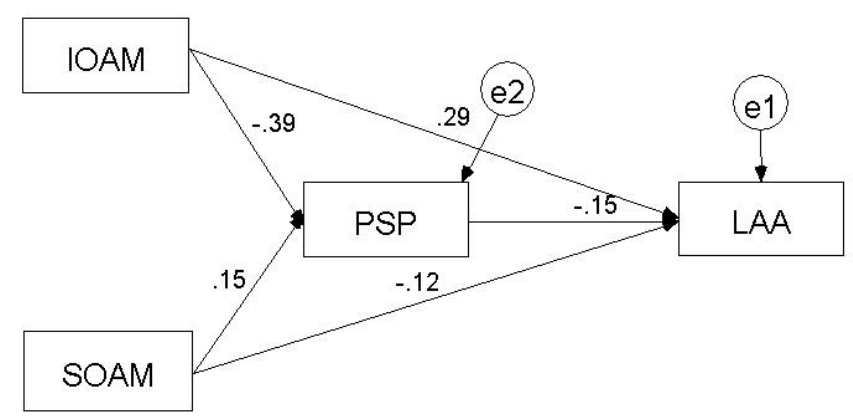

Figure 1: The mediation of study pressure: the effect of IOAM and SOAM on academic performance.

The analysis of the reduced-form model showed that PSP partially mediated the relationship between IOAM, SOIM and LAA. The total effect (c1) of IOAM on LAA was 0.343 $(\mathrm{SE}=0.10,95 \% \mathrm{CI}[0.301,0.356])$, and the total effect (c2) of SOAM on LAA was -0.141 ( $\mathrm{SE}=0.10$, 95\%CI [-0.152, $-0.123])$. IOAM had a direct effect ( $1^{\prime}$ ') of 0.29 ( $\mathrm{SE}=0.10$, 95\%CI [0.25, 0.34]) on LAA, SOAM' direct effect (c2') was $-0.12(\mathrm{SE}=0.10,95 \% \mathrm{CI}[-0.21,-0.06])$ on LAA. The standardized indirect effects of IOAM and SOAM were 0.057 and -0.022 . The reduced-form model mediation model showed statistical good model fit of data: $\chi 2=1.564, \mathrm{df}=1$, $\chi 2 / \mathrm{df}=1.564, \quad \mathrm{p}=0.000 ; \quad \mathrm{TLI}=0.971 ; \quad \mathrm{CFI}=0.995$; RMSEA=0.042 (90\% CI [0.000, 0.161]; $\mathrm{p}=0.013)$.

\section{Discussion}

The key objective of this study is to explore the effect of SOAM and IOAM on students' academic performance, and examine the mediating role of students' perception of school climate on the relationship between IOAM, SOIM and LAA. Pearson correlation analysis results showed that there is a significant negative correlation between LAA and SOAM, but LAA positively correlated with IOAM, which is similar to previous study. Elliot, McGregor and Gable (1999) found that performance-approach goals are positive predictors of exam performance, while predicative effect of performance-avoidance goals is negative. These results indicate that different goal orientations have different predictive effects on students' academic performances. In addition, we found that the perception of study pressure, school order and discipline are negatively correlated with academic performance, while the perception of teacher-student relationship, classmate relationship and the development of diversity are positively correlated with academic performance.

The results of mediation analysis showed that SOAM and IOAM have direct effect on students' academic performances. However, SOAM has negative effects on the academic performance; while IOAM predict positively the academic performance. These results support partially our research hypotheses that there is difference in the effect of SOAM and IOAM on the academic performance. Moreover, not all aspects of school climate perception mediate the effect of achievement motivation on academic performance, and the perception of study pressure has a partially mediating effect in the relationship between both motivation orientations and the academic performance. The perception of study pressure predicts negatively students' academic performance, and SOAM predict positively the perception of study pressure; while IOAM predict negatively the perception of study pressure.

The vast majority of studies believed that teacher-student relationships, classmate relationship were predictors of students' academic outcomes (e.g. academic skills, academic performance, behavioral engagement, etc. al. (Košir, \& Tement, 2014; Lee, 2012; Lubbers, Van Der Werf, Snijders, Creemers, \& Kuyper, 2006). For example, there were some studies found that positive teacher-student relationships could improve academic skills in students as early as middle school and as late as high school (Midgley et al., 1989; Murray \& Malmgren, 2005), and students who were accepted by their peers had lower probabilities to retain a grade or to move downward in the track system (Lubbers, et al., 2006). Furthermore, $\mathrm{Yu}$ and $\mathrm{Ge}$ (2010) found that students' perception of the development of diversity was also predictor positively their academic performance, which provides support for the correlation analysis results in the present study. Therefore, we should pay attention to positive teacher-student relationship and classmate relationship, and create a caring, accepting, and friendly school climate and provide opportunities of diversified developments for students, which will improve students' learning engagement and academic performance.

On the other hand, Pearson correlation analysis results showed students' academic performance are negatively correlated with their perception about study pressure, school order and discipline. The result about study pressure is consistent with $\mathrm{Yu}$ and Ge (2010). They found that study pressure have negatively relation with academic performance, while it is not supported by the Lee's (2012) research results that there is a positive correlation between study pressure and academic achievement. It might be because that there is difference in the definition about study pressure. Lee (2012) used "academic pressure" that refers to "demandingness" which was measured by students' perceptions about the existence of pressure toward academic excellence, and he believed that academic pressure "can be also considered as a form of teacher support" in a sense. In our study, study pressure is student's subjective feelings about learning tasks and study schedules. Positive support and expectations help to create a positive campus atmosphere, which leads to a positive correlation between academic pressure and academic achievement (Lee, 2012; Ma, 2003). However, if students experience excessive learning tasks and more urgent learning time, it may lead to negative learning behaviors and academic outcomes. Similarly, school order and discipline were originally used to maintain a good teaching environment, but if students experience more constraints and control over them, it may also lead to negative academic outcomes. 
Students' motivation to learning is often mediated by their perception of school climate. For example, students who perceive their relationship with their teachers, classmates as positive, warm and close are motivated to improve their academic achievement (Hughes, Cavell, \& Willson, 2001; Lubbers, et al,. 2006). Conversely, if they perceive rejection and exclusion from their school climate, then their learning engagement may be reduced, and their academic performance will fall (Greenman, Schneider, \& Tomada, 2009). In this study, we found that there is only one factor (perception of study pressure) mediates the relationship between achievement motivation and academic performance. For students with IOAM, they have clear personal goals and confidence to realize their goals through hard working; however, when they perceived more learning tasks and study pressure from others, it would make them believe that their personal achievement goals are less likely to be realized, which leads to them lose learning interest and to decrease their academic performance. Furthermore, the academic performances of students who are SOAM would be poor because they lack intrinsic motivation, and when they perceived greater pressure of learning, they would tend to reduce interactions with their classmates and teachers, so that they would not experience the positive expectations, warm, and caring from others (Birch, \& Ladd, 1998; Košir, \& Tement, 2014), and their academic performance might be declined. Therefore, school education would guide students to clarify their learning goals, reduce external study pressure, and create positive school environment.

This study has several limitations. First, this study only used the questionnaire survey, and all data were collected from participants' self-report, which may not fully reflect their achievement motivation orientation and the perception about different aspects of school climate. In the future research, the interview and the experimental methods would be used to explore students' achievement motivation, perception of school climate and its effect on their learning. Second, in this study, the index of students' academic performance is the average scores of Chinese, mathematics, and English. In fact, different students may have different learning attitudes and engagement for different subjects. So, we need further explore the correlation on a particular curriculum. There are researches that explored relations between mathematics achievement, English achievement and motivation (Ma, Du, Hau, \& Liu, 2017; Prast, Van de Weijer-Bergsma, Miočević, Kroesbergen, \& Van Luit, 2018). Third, there are several factors that affect students' academic performance, such as individuals' cognitive ability, self-regulation ability, family income, parents' style, etc (Aunola, Stattin, \& Nurmi, 2000; Bücker, Nuraydin, Simonsmeier, Schneider, \& Luhmann, 2018; Kriegbaum, Becker, \& Spinath, 2018; Murray, \& Malmgren, 2005). It is necessary to effectively control these variables in the future study. Furthermore, further study need pay attention to Chinese students' value belief diversities in the process of globalization and their age variations (Košir, \& Tement, 2014).

\section{Acknowledgements}

This work was supported by the 2021 Pedagogy project of National Social Science Foundation in "Individual Psychological Construction and Education Promotion
Strategy of the Sense of Community for the Chinese Nation" (Grant No. BBA210041).

\section{References}

[1] Ames, C. A. (1990). Motivation: What teachers need to know. Teachers College Record, 91(3), 409-421.

[2] Amemiya, J., \& Wang, M. T. (2018). African American adolescents' gender and perceived school climate moderate how academic coping relates to achievement. Journal of School Psychology, 69, 127-142.

[3] Aunola, K., Stattin, H., \& Nurmi, J. E. (2000). Parenting styles and adolescents' achievement strategies. Journal of Adolescence, 23, 205-222.

[4] Bipp, T., \& van Dam, K. (2014). Extending hierarchical achievement motivation models: The role of motivational needs for achievement goals and academic performance. Personality and Individual Differences, 64, 157-162.

[5] Birch, S. H., \& Ladd, G. W. (1998). Children's interpersonal behavior and the teacher-child relationship. Developmental Psychology, 34, 934-946.

[6] Bond.R. M., A Chykina, V., \& Jones, J. J. (2017). Social network effects on academic achievement. The Social Science Journal, 54(4), 438-449.

[7] Briley, D., Wyer, R. S., \& Li, E. (2014). A dynamic view of cultural influence: A review. Journal of Consumer Psychology, 24(4), 557-571.

[8] Bücker, S., Nuraydin, S., Simonsmeier, B. A., Schneider, M., \& Luhmann, M. (2018). Subjective well-being and academic achievement: A meta-analysis. Journal of Research in Personality, 74, 83-94.

[9] Bush, T., \& Haiyan, Q. (2000). Leadership and culture in Chinese education. Asia Pacific Journal of Education, 20(2), 58-67.

[10] Cohen, G. L., Garcia, J., \& Goyer, J. P. (2017). Turning point: Targeted, tailored, and timely psychological intervention. Handbook of competence and motivation.

[11] De Pedro, K. T., Gilreath, T., Berkowitz, R. (2016). A latent class analysis of school climate among middle and high school students in California public schools. Children and Youth Services Review, 63, 10-15.

[12] Elliot, A. J. Mcgregor, H. A., \& Gable, S. L.. (1999). Achievement goals, study strategies, and exam performance: A mediational analysis. Journal of Educational Psychology, 91(3), 549-563.

[13] Englund, M. M., Luckner, A. E., Whaley, G. J. L., \& Egeland, B. (2004). Children's achievement in early elementary school: Longitudinal effects of parental involvement, expectations, and quality of assistance. Journal of Educational Psychology, 96(4), 723-730.

[14] Greenman, P. S., Schneider, B. H., \& Tomada, G. (2009). Stability and change in patterns of peer rejection: Implications for children's academic performance over time. School Psychology International, 30, 163-183.

[15] Hu, L., \& Bentler, P. M. (1999). Cutoff criteria for fit indexes in covariance structure analysis: Conventional criteria versus new alternatives. Structural Equation Modeling, 6, 1-55.

[16] Hughes, J. N., Cavell, T. A., \& Willson, V. (2001). Further support for the developmental significance of the quality of the teacher-student relationship. Journal of School Psychology, 39(4), 289-301. 
[17] Jelas, Z. M., Azman, N., Zulnaidi, H., \& Ahmad, N. A. (2016). Learning support and academic achievement among Malaysian adolescents: The mediating role of student engagement. Learning Environments Research, 19(2), 221-240.

[18] Košir, K., \& Tement, S. (2014). Teacher-student relationship and academic achievement: A cross-lagged longitudinal study on three different age groups. European Journal of Psychology of Education, 29(3), 409-428.

[19] Koth, C.W., Bradshaw, C. P., \& Leaf, P. J. (2008). A multilevel study of predictors of student perceptions of school climate: The effect of classroom-level factors. Journal of Educational Psychology, 100(1), 462-473.

[20] Kriegbaum, K., Becker, N., \& Spinath, B. (2018). The relative importance of intelligence and motivation as predictors of school achievement: A meta-analysis. Educational Research Review, 25, 120-148.

[21] Kuperminc, G. P., Leadbeater, B. J., Emmons, C., \& Blatt, S. J. (1997). Perceived school climate and difficulties in the social adjustment of middle school students. Applied Developmental Science, 1, 76-88.

[22] Kuperminc, G. P., Leadbeater, B. J., \& Blatt, S. J. (2001). School social climate and individual differences in vulnerability to psychopathology among middle school students. Journal of School Psychology, 39, 141-159.

[23] Lee, J. S. (2012). The effects of the teacher-student relationship and academic press on student engagement and academic performance. International Journal of Educational Research, 53, 330-340.

[24] Loukas, A., \& Robinson, S. (2004). Examining the moderating role of perceived school climate in early adolescent adjustment. Journal of Research on Adolescence, 14, 209-233.

[25] Lubbers, M. J., Van Der Werf, M. P. C., Snijders, T. A. B., Creemers, B. P. M., \& Kuyper, H. (2006). The impact of peer relations on academic progress in junior high. Journal of School Psychology, 44(6), 491-512.

[26] Ma, L. H., Du, X. F., Hau, K. T., \& Liu, J. (2017). The association between teacher-student relationship and academic achievement in Chinese EFI context: A serial multiple mediation model. Educational Psychology, 38(5), 687-707.

[27] Ma, X. (2003). Sense of belonging to school: Can schools make a difference? Journal of Educational Research, 96, 340-349.

[28] Mcinerney, D. M., Maehr, M. L., \& Dowson, M. (2004). Motivation and culture. Encyclopedia of Applied Psychology, 2, 631-639.

[29] Midgley, C., \& Eccles, F. J. S. (1989). Student/teacher relations and attitudes toward mathematics before and after the transition to junior high school. Child Development, 60(4), 981-992.

[30] Modin, B., \& Östberg, V. (2009). School climate and psychosomatic health: A multilevel analysis. School Effectiveness and School Improvement, 20(4), 433-455.

[31] Murray, C., \& Malmgren, K. (2005). Implementing a teacher-student relationship program in a high-poverty urban school: Effects on social, emotional, and academic adjustment and lessons learned. Journal of School Psychology, 43(2), 137-152.

[32] Nelson, R. M., \& DeBacker, T. K. (2008). Achievement motivation in adolescents: The role of peer climate and best friends. The Journal of Experimental Education, 76(2), 170-189.

[33] Nickerson, A., Singleton, D., Schnurr, B., \& Collen, M. (2014). Perceptions of school climate as a function of bullying involvement. Journal of Applied School Psychology, 30(2), 157-181.

[34] Prast, E. J., Van de Weijer-Bergsma, E. Miočević, M. Kroesbergen, E. H., \& Van Luit, J. E.H. (2018). Relations between mathematics achievement and motivation in students of diverse achievement levels. Contemporary Educational Psychology, 55, 84-96.

[35] Purkey, S., \& Smith, M. (1983). Effective schools: A review. The Elementary School Journal, 83, 427-452.

[36] Reindl, M., Berner, V. D., Scheunpflug, A., Zeinz, H., \& Dresel, M. (2015). Effect of negative peer climate on the development of autonomous motivation in mathematics. Learning \& Individual Differences, 38, 68-75.

[37] Ryan, A. M., \& Patrick, H. (2001). The classroom social environment and changes in adolescents' motivation and engagement during middle school. American Educational Research Journal, 38, 437-460.

[38] Roberts, G. C. (2001). Advances in motivation in sport and exercise. Champaign, IL: Human Kinetics

[39] Sommer, B. (1985). What's different about truants? A comparison study of eighth graders. Journal of Youth and Adolescence, 14, 411-422.

[40] Tao, V. Y. K., \& Hong, Y. (2000). A meaning system approach to Chinese students' achievement goals. Journal of Psychology in Chinese Societies, 1, 13-38.

[41] Tao, V. Y. K., \& Hong, Y. (2013). When academic achievement is an obligation: perspectives from social-oriented achievement motivation. Journal of Cross-Cultural Psychology, 45(1), 110-136.

[42] Thapa, A., Cohen, J., Guffey, S., \& Higgins-D'Alessandro, A. (2013). A review of school climate research. Review of Educational Research, 83(3), 357-385.

[43] Theodosiou, A., \& Papaioannou, A. (2006). Motivational climate, achievement goals and metacognitive activity in physical education and exercise involvement in out-of-school settings. Psychology of Sport and Exercise, 7, 361-379.

[44] Wagner, J. A. (2002). Utilitarian and ontological variation in individualism-collectivism. Research in Organizational Behavior, 24(3), 301-345.

[45] Wang, M. T., \& Holcombe, R. (2010). Adolescents' perceptions of school environment, engagement, and academic achievement in middle school. American Educational Research Journal, 47(3), 633-662.

[46] Wentzel, K.R., Battle, A., Russell, S.L., \& Looney, L.B. (2010). Social supports from teachers and peers as predictors of academic and social motivation. Contemporary Educational Psychology, 35(3), 193-202.

[47] Vandenberg, R.J., \& Lance, C. E. (2000). A review and synthesis of the measurement invariance literature: Suggestions, practices, and recommendations for organizational research. Organizational Research Methods, 3(1), 4-70.

[48] Viljaranta, J., Aunola, K., \& Hirvonen, R. (2016). Motivation and academic performance among first-graders: A person-oriented approach. Learning and Individual Differences, 49, 366-372. 
[49] Yu, A.-B., \& Yang, K.-S. (1994). The nature of achievement motivation in collectivist societies. In U. Kim, H. C. Triandis, C. Kagitcibasi, S.-C. Choi, \& G. Yoon (Eds.), Individualism and collectivism: Theory, method, and applications (pp. 235-239). Newbury Park, CA: SAGE.

[50] Zhang,Y. Y., Qin, X. N., \& Ren, P.(2018). Adolescents' academic engagement mediates the association between Internet addiction and academic achievement: The moderating effect of classroom achievement norm. Computers in Human Behavior, 89, 299-307.

[51] Zullig, K., Koopman, T., Patton, J., \& Ubbes, V. (2010). School climate: Historical review, instrument development, and school assessment. Journal of Psychoeducational Assessment, 28, 139-152. 